\title{
Primary lymphoepithelioma-like carcinoma of the lung: An unusual cancer and clinical outcomes of 14 patients
}

\author{
LAN LIN $^{1}$, TINGYAN LIN ${ }^{1}$ and BANGWEI ZENG ${ }^{2}$ \\ Departments of ${ }^{1}$ Respiratory Medicine and ${ }^{2}$ Hospital Infection Management, Fujian \\ Medical University Union Hospital, Fuzhou, Fujian 350001, P.R. China
}

Received April 30, 2016; Accepted April 13, 2017

DOI: $10.3892 / \mathrm{ol} .2017 .6510$

\begin{abstract}
Advanced lung cancer is considered to exhibit a poor prognosis; however, the pulmonary lymphoepithelioma-like carcinoma (LELC), a rare subtype of non-small cell lung cancer (NSCLC), exhibits an improved prognosis, compared with non-LELC. The present study aimed at investigating the clinical manifestation, imaging characteristics, pathology, tumor markers, treatment and prognosis of primary LELC of the lung. A total of 14 patients with pulmonary LELC were confirmed by surgery and pathology. Clinical data of those patients were retrospectively reviewed including age, sex, smoking history, symptoms, computed tomography (CT) results, Epstein-Barr virus-encoded RNA (EBER) status, treatment and outcomes. In the present study, there were 7 males and 7 females who ranged in age between 22 and 64 years (mean, 51.21 \pm 11.37 years) and who all were from eastern China. The tumor-node-metastasis stage ranged between stages I and IV, with $71.43 \%$ of the patients at advanced stage (stages III and IV). The results of the present study identified $100 \%$ positive expression of EBER. Tumors located centrally were of significantly increased size, compared with peripheral tumors $(\mathrm{P}<0.05)$, and lymphadenopathy was more common in patients with advanced stage $(\mathrm{P}<0.05)$. The majority of patients were treated with surgery, platinum-based chemotherapy or radiotherapy. At time of writing, 12 patients were alive and the longest survival time was 60 months. Pulmonary LELC typically affected young patients and was not associated with smoking history; however, pulmonary LELC was associated with Epstein-Barr virus infection in the Asian population. The majority of patients were in early or locally advanced stages and exhibit an improved prognosis compared with other types of NSCLC. Pulmonary LELC was sensitive to chemotherapy
\end{abstract}

Correspondence to: Miss Lan Lin, Department of Respiratory Medicine, Fujian Medical University Union Hospital, 29 Xinquan Road, Fuzhou, Fujian 350001, P.R. China

E-mail: linlan3735882@163.com

Key words: lymphoepithelioma-like carcinoma, Epstein-Barr virus, treatment, prognosis and surgery, with postoperative chemotherapy-based multimodality treatment recommended.

\section{Introduction}

Lymphoepithelioma-like carcinoma (LELC) is a rare type of cancer and typically occurs in pharyngeal and foregut-derivative organs, including the salivary glands, thymus, stomach and liver (1). However, cases of LELC in additional anatomical locations have been previously reported (2-6), including the bladder, lacrimal glands, ovaries, cervix and skin. Primary LELC of the lung, which is a subtype of non small cell lung cancer (NSCLC) (1), was first studied by Begin et al (7) in 1987 and, compared with other type of NSCLC, patients with primary LELC of the lung are rare. Primary LELC carries an improved clinical outcome compared with other types of NSCLC, even with advanced stages of the disease (1). Pulmonary LELC typically affects the young and non-smoking population, and is associated with Epstein-Barr virus (EBV) infection. Over the last two decades, 150 cases of lung LELC have been reported in the literature worldwide (1). However, a limited number of studies have specifically described the typical clinical and pathological characteristics, computed tomography (CT) results and therapy of the disease. Available treatments of pulmonary LELC include surgical resection, chemotherapy and radiotherapy, according to the different stages of the tumor (1). Furthermore, the optimal treatment and long-term follow-up results were limited due to the low rate of incidence. Therefore, the present retrospective study accessed the databases of Fujian Medical University Union Hospital to review all patients who were diagnosed with pathologically confirmed pulmonary LELC between June 2010 and June 2014. The present study aimed at determining the aforementioned information, including clinical manifestation, imaging characteristics, pathology, tumor markers, Epstein-Barr virus-encoded RNA (EBER) status, treatment and outcomes, in order to understand this disease and identify an optimal treatment method.

\section{Materials and methods}

Subjects. The present retrospective study collected the clinical data of 14 cases of patients with pathologically proven pulmonary LELC between June 2010 and June 2014 
in Fujian Medical University Union Hospital (Fujian, China). All procedures were approved by the Ethics Committee of the Fujian Medical University Union Hospital (Fujian, China) and written informed consent was obtained from all patients. Focus was placed on the patients' sex, age, symptoms, smoking status, tumor staging, CT results, treatments and outcome. All patients were initially diagnosed at the Fujian Medical University Union Hospital, 7 patients were male, 7 patients were female and age ranged between 22 and 64 years (mean, $51.21 \pm 11.37$ years). All patients underwent a chest CT scan, blood biochemistry and blood tumor marker test, and 4 patients accepted a positron emission tomography (PET)-CT scan.

Pathological diagnosis. All patients were diagnosed by histopathology; 12 cases were confirmed following surgery and 2 cases were diagnosed using superficial lymph node biopsy. Tumor tissues were determined using routine hematoxylin-eosin (H\&E) staining, immunohistochemistry and Epstein-Barr virus-encoded RNA (EBER) test. All cases were diagnosed according to the diagnostic standard of pulmonary LELC (8). Clinical staging was assessed using the 2009 International Tumor-Node-Metastasis staging of lung cancer (9).

Therapy. In the present study, 2 cases received only surgery, 9 cases received surgery and chemotherapy, 1 case received surgery, chemotherapy and radiotherapy, and 2 cases, classified as stage IIIB following a superficial lymph node biopsy, were discharged from hospital without any treatment. The 12 patients who underwent surgery received the following: 8 patients, classified between stage IA and IIIB, received a pulmonary lobectomy and systematic lymphadenectomy; 2 patients (stage IIIA and stage IIIB) underwent a pure resection of pulmonary tumor; 1 patient, stage IV, received a right side thoracoscopy, pleural biopsy and pleurodesis; and the final patient, stage IV, received a wedge-shape excision of lung under video-assisted thoracoscopic surgery and pleural biopsy, since the chest wall was involved. The patients received platinum-based chemotherapy combined with paclitaxel, pemetrexed, docetaxel, navelbine or gemcitabine which was repeated every 3 or 4 weeks for a total of 4 or 5 cycles. One of the patients underwent a CT scan following the third course of chemotherapy which revealed that the paratracheal lymph nodes had increased in size by $10 \%$, therefore the patient was administered concurrent radiochemotherapy. Following two cycles of chemotherapy, all patients received a chest CT scan to evaluate the curative effect.

Follow-up visits. One patient with stage IV LELC could not be contacted 4 months following diagnosis. The remaining 13 patients' follow-up information, between 12 and 60 months after diagnosis (until 30 July 2015), was obtained from the patients' medical records, the outpatient department or by a telephone interview with the patients. All patients, except for one, survived during the follow-up period; the longest survival time was $\geq 60$ months, the shortest survival time was 14 months and the median survival was $35.35 \pm 17.58$ months.

Statistical analysis. Statistical analysis was performed using SPSS software (version 19.0; IBM Corp., Armonk, NY, USA). Wilcoxon's signed rank test was used to determine significant differences between tumor sizes. Fisher's exact test was used to determine the association between tumor stage and lymphadenopathy. Spearman's correlation coefficient was used to determine the association between maximum diameter of tumor and serum levels of the tumor marker cytokeratin-19 fragments (Cyfra21-1). All results are presented as the mean \pm standard deviation. $\mathrm{P}<0.05$ was considered to indicate a statistically significant difference.

\section{Results}

Clinical results. Tables I and II present the clinical information and characteristics of 14 patients; 7 of which were male and 7 were female. The age of the patients ranged between 22 and 64 years with a mean age of $51.21 \pm 11.37$ years and a median age of 54.5 years. All patients were from Fujian (China) and $28.57 \%$ of patients in the present cohort had a history of smoking. A total of 9 patients suffered from a cough, expectoration, chest pain, hemoptysis or fever between 3 days and 1 year; however, the remaining 5 patients exhibited no symptoms. Of the patients in the present study, 4 were at an early stage (stages I and II) and the remaining 10 were at an advanced stage (stages III and IV).

Imaging characteristics. A total of 14 patients underwent a chest CT scan at initial diagnosis. CT scans suggested that LELC of the lung exhibited the following characteristics (Table III): Typically a single lesion; primarily of a peripheral location (accounting for $78.57 \%$ of cases); large size (maximum diameter between 1.6 and $6.5 \mathrm{~cm}$, mean diameter of $3.91 \pm 1.69 \mathrm{~cm}$ ), inhomogeneous density; lobular or spiculated signs; pleural indentation; cavitation; liquefactive necrosis; calcification; compression of the vena cava; bronchiarctia; and lymphadenopathy. In the present study, 2 patients exhibited metastatic spread to the pleural space and 1 patient exhibited lung-to-lung metastasis. Distinct CT manifestations are presented in Figs. 1-3. Comparisons were made between tumor sizes depending on tumor localization, tumor stage and enhancement pattern (Table IV). The results of the present study identified that central tumors appeared to be larger than peripheral tumors $(\mathrm{P}=0.027)$. The association between lymphadenopathy and tumor stage was analyzed and it was demonstrated that lymphadenopathy was significantly more common in patients at an advanced stage $(\mathrm{P}=0.041)$.

A total of 4 patients underwent a PET-CT scan (Fig. 4) which revealed that radioactive uptake increased in lobular or spiculated lesions exhibiting high metabolic soft tissue density shadow, a slightly rough edge, inhomogeneous density and pleural indentation, with a maximum standard uptake value $\left(\mathrm{SUV}_{\max }\right)$ ranging between 5.4 and 11.6.

Blood results. Serum tumor makers, including carcinoembryonic antigen (CEA) and Cyfra21-1, albumin (ALB) and lactate dehydrogenase $(\mathrm{LDH})$ were determined during pretreatment clinical evaluation (Table V). Serum levels of CEA and LDH increased in 1 patient $(1 / 14,7.14 ; 1 / 14,7.14 \%$, respectively), Cyfra21-1 increased in 6 patients $(6 / 13,46.15 \%)$, whereas ALB decreased in 2 patients $(2 / 14,14.29 \%)$. Statistically, the correlation coefficient of the maximum tumor diameter and Cyfra21-1 was $0.696(\mathrm{P}=0.008)$. 
Table I. Clinical information of 14 patients with pulmonary LELC.

\begin{tabular}{|c|c|c|c|c|c|c|c|}
\hline $\begin{array}{l}\text { Case no./ } \\
\text { sex/age, } \\
\text { years }\end{array}$ & $\begin{array}{c}\text { Smoking } \\
\text { history }\end{array}$ & $\begin{array}{r}\text { Tumor } \\
\text { location }\end{array}$ & $\begin{array}{l}\text { Size } \\
\mathrm{cm}\end{array}$ & $\begin{array}{l}\text { Stage } \\
(\mathrm{TNM})\end{array}$ & EBER & Treatment & $\begin{array}{c}\text { Outcome (Alive/ } \\
\text { Deceased/Could not be } \\
\text { contacted since diagnosis) }\end{array}$ \\
\hline $1 / \mathrm{M} / 38$ & Yes & RUL & $3.5 \times 3.4$ & IIIB (T2a N3 M0) & + & No treatment & Alive, 20 months \\
\hline 2/M/56 & Yes & LUL & $1.6 \times 1.2$ & IIIA (T2 N2 M0) & ND & $\mathrm{SG}+\mathrm{ChT}(\mathrm{TP} \times 5)$ & Alive, 60 months \\
\hline $3 / \mathrm{F} / 61$ & No & RUL & $6.3 \times 6.1$ & IIIB (T4 N2 M0) & ND & SG & Alive, 54 months \\
\hline 4/M/55 & No & LLL & $5.9 \times 3.6$ & IIB (T2b N1 M0) & ND & $\mathrm{SG}+\mathrm{ChT}(\mathrm{TP} \times 4)$ & Alive, 58 months \\
\hline $5 / F / 52$ & No & RLL & $6.5 \times 5.0$ & IV (T2 N1 M1a) & + & Biopsy + ChT (TP x4) & Alive, 17 months \\
\hline $6 / F / 22$ & No & RML & $2.1 \times 2.0$ & IIIB (T2 N3 M0) & + & $\mathrm{SG}+\mathrm{ChT}(\mathrm{NP} \times 4)+\mathrm{RT}$ & Alive, 22 months \\
\hline 7/M/61 & No & LUL & $2.0 \times 2.0$ & IIIA (T1a N2 M0) & + & $\mathrm{SG}+\mathrm{ChT}(\mathrm{PP} \times 5)$ & Alive, 25 months \\
\hline 8/M/57 & Yes & RLL & $4.6 \times 4.0$ & IV (T2a N3 M1) & + & No treatment & Deceased, 14 months \\
\hline 9/M/61 & No & RML & $3.5 \times 3.0$ & IB (T2a N0 M0) & + & $\mathrm{SG}+\mathrm{ChT}(\mathrm{DP} \times 4)$ & Alive, 39 months \\
\hline $10 / \mathrm{F} / 64$ & No & LLL & $2.3 \times 1.9$ & IA (T1 N0 M0) & + & SG & Alive, 38 months \\
\hline $11 / \mathrm{M} / 46$ & Yes & RML & $3.5 \times 3.0$ & IB (T2a N0 M0) & + & $\mathrm{SG}+\mathrm{ChT}(\mathrm{DP} \times 4)$ & Alive, 31 months \\
\hline $12 / F / 42$ & No & RUL & $6.0 \times 5.1$ & IIIA (T4 N0 M0) & ND & $\mathrm{SG}+\mathrm{ChT}(\mathrm{NP} \times 1+\mathrm{GP} \times 3)$ & Alive, 12 months \\
\hline $13 / F / 54$ & No & LLL & $3.4 \times 2.7$ & IIIA (T2a N2 M0) & ND & $\mathrm{SG}+\mathrm{ChT}(\mathrm{GP} \times 4)$ & Alive, 31 months \\
\hline $14 / F / 48$ & No & LLL & $3.6 \times 2.7$ & IV (T2 N2 M1a) & + & $\mathrm{SG}+\mathrm{ChT}(\mathrm{TP} \times 4)$ & Could not be contacted \\
\hline
\end{tabular}

TNM, tumor-node-metastasis; F, female; M, male; RUL, right upper lobe of lung; LUL, left upper lobe of lung; LLL, left lower lobe of lung; RLL, right lower lobe of lung; RML, right middle lobe of lung; +, positive; ND, not done; SG, surgery; ChT, chemotherapy; RT, radiotherapy; EBER, Epstein-Barr virus-encoded RNA; TP, paclitaxel + cisplatin/carboplatin; NP, navelbine + cisplatin/carboplatin; DP, docetaxel + cisplatin/carboplatin; PP, pemetrexed + cisplatin/carboplatin; GP, gemcitabine + cisplatin/carboplatin; LELC, lymphoepithelioma-like carcinoma.

Table II. Clinical characteristics of patients with pulmonary LELC.

\begin{tabular}{|c|c|}
\hline Variable & $\mathrm{n}(\%)$ \\
\hline \multicolumn{2}{|l|}{ Sex } \\
\hline Male & $7(50.0)$ \\
\hline Female & $7(50.0)$ \\
\hline \multicolumn{2}{|l|}{ Age, years } \\
\hline Median & 54.5 \\
\hline Range & $22-64$ \\
\hline$<60$ & $10(71.43)$ \\
\hline$\geq 60$ & $4(28.57)$ \\
\hline \multicolumn{2}{|l|}{ Smoking status } \\
\hline Former or current smoker & $4(28.57)$ \\
\hline Non-smoker & $10(71.43)$ \\
\hline \multicolumn{2}{|l|}{ Stage } \\
\hline IA/IB & $1 / 2(7.14 / 14.29)$ \\
\hline IIA/IIB & $0 / 1(0 / 7.14)$ \\
\hline IIIA/IIIB & $4 / 3(28.57 / 21.43)$ \\
\hline IV & $3(21.43)$ \\
\hline \multicolumn{2}{|l|}{ Symptom } \\
\hline Cough, expectoration & $4(28.57)$ \\
\hline Chest pain & $2(14.29)$ \\
\hline Hemoptysis & $2(14.29)$ \\
\hline Fever & $1(7.14)$ \\
\hline
\end{tabular}

LELC, lymphoepithelioma-like carcinoma.
Pathological results. On visual inspection, pulmonary LELC typically appeared as round or oval nodules, medium or hard texture and gray section, and were primarily accompanied by hemorrhage or necrosis. Microscopic observation revealed large clusters of cancer cells with round or oval shape, large nuclei, hyperchromatic, irregular contour, prominent nucleoli and prominent lymphocyte infiltration in the interstitial substance. An EBER test was carried out in 9 cases; all of which were positive.

Treatment outcome. Chest CT scans were examined to evaluate the effects of chemotherapy and these revealed that all cases obtained a partial response. At the time of writing, all except 1 of the 12 patients who accepted surgery or chemotherapy or radiotherapy, survived. Of the 12 patients who received follow-up, 10 exhibited tumor-free survival (2 patients survived $>1$ year, 3 patients survived $>2$ years and 5 patients survived $>3$ years) and 1 patient survived with a tumor. The longest survival time was 60 months.

\section{Discussion}

Primary pulmonary LELC is a rare malignant tumor which accounts for only $0.9 \%$ of all primary lung cancer $(10,11)$ and only represents $0.4 \%$ of NSCLC (12). Primary LELC of the lung is a subtype of large cell carcinoma of the lung, according to the World Health Organization histological typing of lung and pleural tumors (13). Primary pulmonary LELC occurs primarily in young and non-smoking Asians; however, $>25 \%$ of the patients included in the present study were $>60$ years old $(4 / 14 ; 28.57 \%)$. Furthermore, there appears to be no differences 
Table III. CT characteristics in 14 patients with primary LELC of the lung.

\begin{tabular}{|c|c|}
\hline $\mathrm{CT}$ characteristic & $\mathrm{n}(\%)$ \\
\hline \multicolumn{2}{|l|}{ Tumor site } \\
\hline Peripheral & 11 \\
\hline Central & 3 \\
\hline Right & 8 \\
\hline Upper & $3(21.43)$ \\
\hline Middle & $3(21.43)$ \\
\hline Lower & $2(14.26)$ \\
\hline Left & 6 \\
\hline Upper & $2(14.26)$ \\
\hline Lower & $4(28.57)$ \\
\hline \multicolumn{2}{|l|}{ Tumor size, $\mathrm{cm}$} \\
\hline$\leq 3$ & $4(28.57)$ \\
\hline$>3$ & $10(71.43)$ \\
\hline \multicolumn{2}{|l|}{ Tumor contour } \\
\hline Lobular or spiculated & $12(85.71)$ \\
\hline Well-defined (smooth) & $2(14.26)$ \\
\hline \multicolumn{2}{|l|}{ Tumor density } \\
\hline Homogenous & $8(57.14)$ \\
\hline Heterogeneous & $6(42.86)$ \\
\hline Enhanced CT scan & 12 \\
\hline Mildly-moderately enhanced & $4(33.33)$ \\
\hline Obviously enhanced & $5(41.67)$ \\
\hline Heterogeneously enhanced & $3(25.00)$ \\
\hline \multicolumn{2}{|l|}{ Relationship with pleura } \\
\hline Direct contact with adjacent pleural surface & $2(14.29)$ \\
\hline Not contact with adjacent pleural surface & $12(85.71)$ \\
\hline Pleural indentation & $2(14.29)$ \\
\hline \multicolumn{2}{|l|}{ Internal characteristics } \\
\hline Cavitation & $1(7.14)$ \\
\hline Liquefactive necrosis & $2(14.29)$ \\
\hline Calcification & $1(7.14)$ \\
\hline Compression of the vena cava & $1(7.14)$ \\
\hline Bronchiarctia & $1(7.14)$ \\
\hline Metastatic spread to the pleural space & $2(14.29)$ \\
\hline Lung-to-lung metastasis & $1(7.14)$ \\
\hline Pericardial invasion & $0(0)$ \\
\hline
\end{tabular}

Lymphadenopathy

None

Ipsilateral peribronchial or ipsilateral hilar (N1) $2(14.29)$

Ipsilateral mediastinal or subcarinal (N2) $\quad 5(35.71)$

Contralateral mediastinal or contralateral $3(21.43)$ hilar or superclavicular (N3)

LELC, lymphoepithelioma-like carcinoma; CT, computed tomography.

observed between sexes (14). Primary pulmonary LELC is associated with EBV infection and exhibits similar histological characteristics to those of nasopharyngeal carcinoma (15).

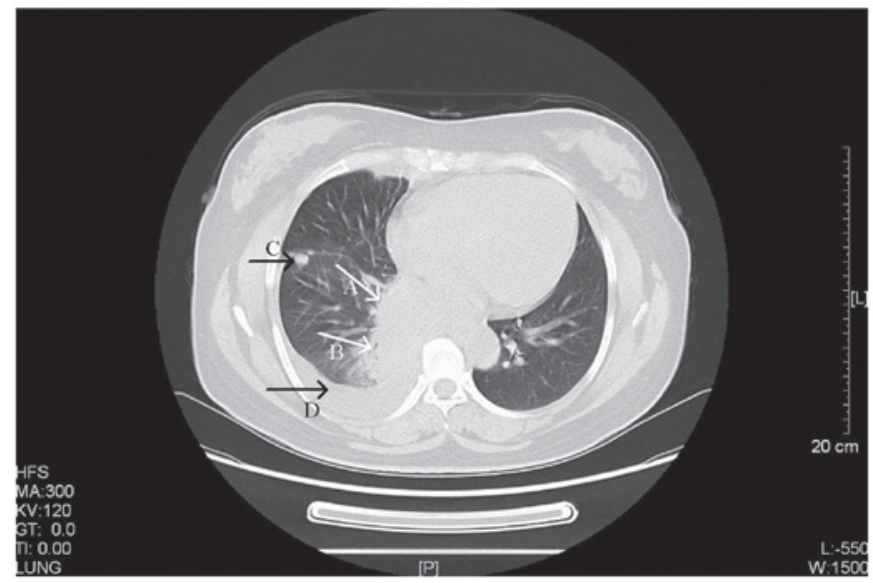

Figure 1. CT scan of a 52-year-old female with stage IV primary pulmonary LELC. Arrow A, a CT scan revealed a large and well-defined soft tissue mass located in the right lower hilus of the lung (size, $6.5 \times 5.0 \mathrm{~cm}$ ). Arrow B, the bronchus in right lower lobe was narrowing with a thickened wall. Arrow $\mathrm{C}$, multiple masses or nodules were observed in the right side of the chest wall, pleura and right oblique fissure with variable sizes (largest, $\sim 1.4 \times 2.7 \mathrm{~cm}$ ). Arrow D, a limited amount of effusion was observed in the right side of the chest. CT, computed tomography; LELC, lymphoepithelioma-like carcinoma. HFS, head first-supine; MA, milliampere; KV, kilovolt; L, windows level; W, windows width; [L], length; GT, gradient; TI, time.

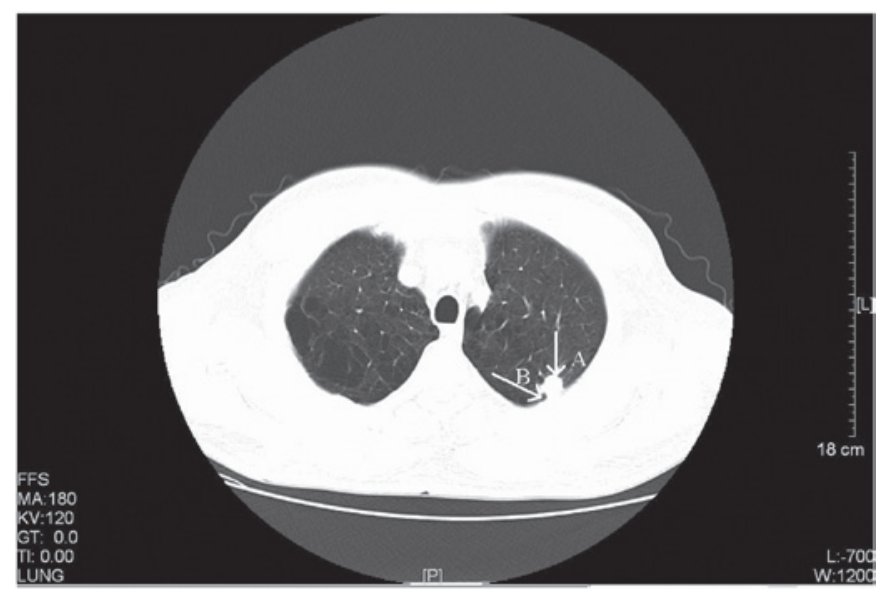

Figure 2. CT scan of a 56-year-old male with stage IIIA primary pulmonary LELC. Arrow A, a CT scan identified a rough-edged, lobular and spiculated nodule in the apicoposterior segmental bronchus of the left upper lobe (size, $1.6 \times 1.2 \mathrm{~cm}$ ). Arrow B, pleural indentation was also observed. CT, computed tomography; LELC, lymphoepithelioma-like carcinoma. FFS, feet first-supine; MA, milliampere; KV, kilovolt; L, windows level; W, windows width; [L], length; GT, gradient; TI, time.

Previous studies have demonstrated that pulmonary LELC is strongly associated with EBV infection in Asians, with $~ 67 \%$ of patients from southern China, Taiwan and Hong Kong $(8,11)$. However, there is no apparent association between EBV infection and pulmonary LELC in the western population (14). The present study retrospectively analyzed a total of 14 patients who were diagnosed with primary pulmonary LELC. All the patients originated from southeast China and there was no marked sex difference in this cohort. Only a limited number of the patients analyzed $(<30 \%)$ had a history of smoking which suggested that primary pulmonary LELC exhibits a minimal association with smoking, in comparison with other 
Table IV. Comparison of tumor size by tumor localization and tumor stage and enhancement pattern.

\begin{tabular}{lcc}
\hline & $\begin{array}{c}\text { Mean } \pm \text { SD tumor size } \\
\text { cm (range) }\end{array}$ & P-value \\
\hline $\begin{array}{l}\text { Tumor localization } \\
\text { Central (n=3) }\end{array}$ & $6.27 \pm 0.25(6-6.5)$ & 0.027 \\
Peripheral (n=11) & $3.27 \pm 1.25(1.6-5.9)$ & \\
Tumor stage & & 0.945 \\
Early (n=4) & $3.80 \pm 1.51(2.3-5.9)$ & \\
Advanced (n=10) & $3.96 \pm 1.83(1.6-6.5)$ & \\
Enhancement pattern & & \\
Homogeneous $(\mathrm{n}=9)$ & $3.69 \pm 1.71(1.6-6.5)$ & \\
Inhomogeneous $(\mathrm{n}=3)$ & $4.87 \pm 2.23(2.3-6.3)$ & \\
\hline
\end{tabular}

$\mathrm{SD}$, standard deviation.

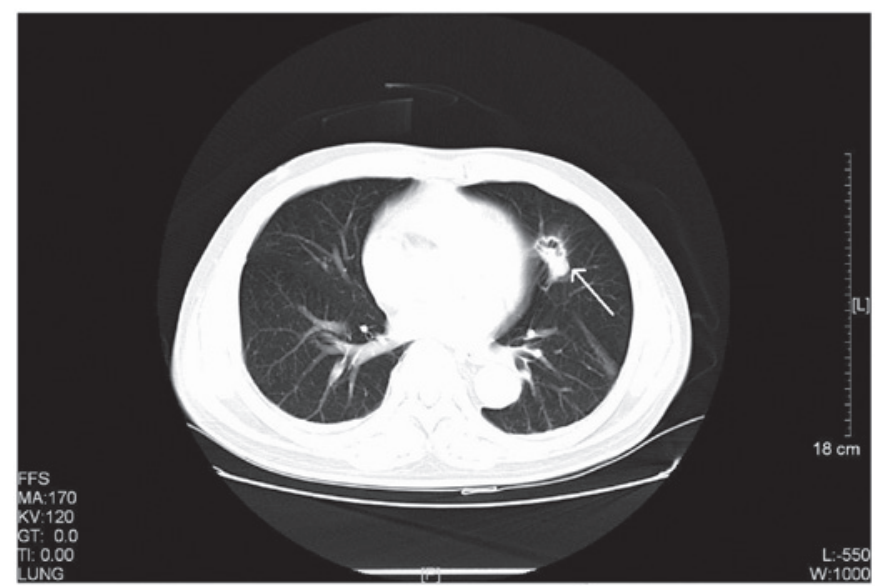

Figure 3. CT scan of a 61-year-old male with stage IIIA primary pulmonary LELC. The CT scan revealed a rough-edged nodule in the lingular segment of the left upper lobe with cavity inside the lesion. CT, computed tomography; LELC, lymphoepithelioma-like carcinoma. FFS, feet first-supine; MA, milliampere; KV, kilovolt; L, windows level; W, windows width; [L], length; GT, gradient; TI, time.

lung cancers. In the present study, in situ hybridization for EBERs was carried out on 9 patient specimens and all were positive, which additionally suggests an association between EBV infection and pulmonary LELC.

To diagnose pulmonary LELC, examination of chest imaging is the first choice, particularly chest CT scans. A previous study (16) hypothesized that advanced pulmonary LELC exhibited the following distinct CT characteristics: Central location, large size, well-defined edges, vascular encasement and peribronchovascular nodal spread. Hoxworth et al (17) suggested that primary pulmonary LELC primarily appears as a peripheral solitary pulmonary nodule close to pleura, $<3.5 \mathrm{~cm}$ in size and typically without lymph node involvement. Ma et al (18) identified that inhomogeneously enhanced tumors were markedly increased in size, compared with homogeneously enhanced tumors $(\mathrm{P}<0.001)$. A previous study (12) demonstrated that direct contact with the adjacent pleural surface was a feature commonly observed on

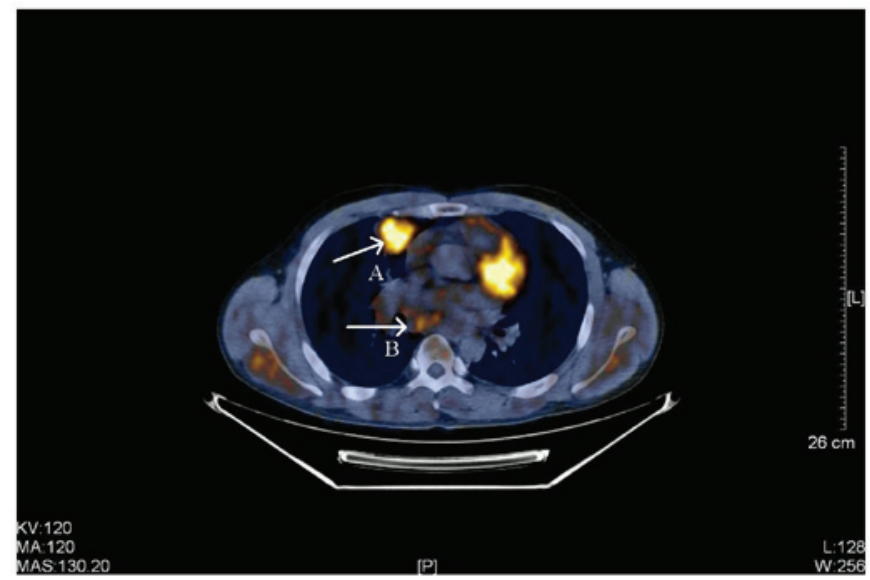

Figure 4. PET-CT scan of a 38-year-old male with stage IIIB primary pulmonary LELC. Arrow A, a PET-CT scan identified a highly metabolic soft tissue (size, $3.5 \times 3.4 \mathrm{~cm})$ with slightly rough edge, lobular, spiculated, inhomogeneous density and pleural indentation in the anterior segment of the right upper lobe. Radioactive uptake increased and the $\mathrm{SUV}_{\max }$ was 5.4. Arrow B, multiple enlarged lymph nodes in the right-side mediastinum and right hilus of the lung. The largest lymph node was located in the mediastinum (size, $2.4 \times 2.2 \mathrm{~cm}$ ), part integrated into clumps and radioactive uptake was slightly increased $\left(\mathrm{SUV}_{\max }, 3.5\right)$. PET-CT, positron emission tomography-computed tomography; LELC, lymphoepithelioma-like carcinoma; $\mathrm{SUV}_{\max }$, maximum standardized uptake value. KV, kilovolt; MA, milliampere; MAS, milliampere sec; L, windows level; W, windows width; [L], length.

CT scans of pulmonary LELC. Therefore, it is hypothesized that doctors suspect pulmonary LELC when solitary peripheral pulmonary nodules with direct contact with the pleural surface in an EBV endemic area are observed.

In the present study, CT scans revealed that pulmonary LELC primarily appears as a single lesion of peripheral location, which is in contrast with previous studies $(16,18)$. Distinctions were observed since the majority of previous studies focused on advanced-stage LELC, whereas patients at early and advanced stage were included in the present study. CT manifestations, including large size, inhomogeneous density, lobulation or spiculation, pleural indentation, cavitation, liquefactive necrosis, compression of the vena cava, bronchiarctia and lymphadenopathy were consistent with previous studies. Central tumors were significantly larger in size compared with that of peripheral tumors $(\mathrm{P}=0.027)$. In addition, CT results of early- and advanced-stage pulmonary LELC demonstrated similarities, with the exception that lymphadenopathy was significantly more common in advanced-stage LELC ( $\mathrm{P}=0.041)$. Lymph node involvement was identified in pulmonary LELC, which was consistent with other types of lung cancer. In the present study, lymph node involvement was identified in $71.4 \%$ (10/14) patients which was similar to results of Ma et al (18) (68.3\%) and decreased compared with results of Ooi et al (16) (100\%). Additionally, the results of the present study identified calcifications in one case and calcifications in solitary pulmonary nodules are typically considered to be evidence of a benign lesion. To the best of our knowledge, pulmonary LELC with calcification has been identified in one previous study, in which contrast CT revealed calcification appeared within necrotic areas; therefore, it was hypothesized that the mechanism of calcification may be dystrophic calcification (18). 
Table V. Patients' blood tumor makers and biochemical results.

\begin{tabular}{|c|c|c|c|c|}
\hline No. & $\begin{array}{c}\text { CEA (normal } \\
\text { value }<5 \mathrm{ng} / \mathrm{ml})\end{array}$ & $\begin{array}{l}\text { Cyfra21-1 (normal } \\
\text { value }<3.3 \mathrm{ng} / \mathrm{ml})\end{array}$ & $\begin{array}{l}\text { ALB (normal value } \\
35-54 \mathrm{~g} / \mathrm{l})\end{array}$ & $\begin{array}{c}\text { LDH (normal } \\
\text { value 109-245 U/l) }\end{array}$ \\
\hline 1 & 0.26 & 5.55 & 41.9 & 163 \\
\hline 2 & 1.6 & 2.48 & 37.4 & 136 \\
\hline 3 & 2.0 & 3.8 & 36.5 & 140 \\
\hline 4 & 4.2 & 7.12 & 37.8 & 162 \\
\hline 5 & 1.7 & 7.07 & 36.1 & 170 \\
\hline 6 & 0.5 & 1.85 & 36.3 & 206 \\
\hline 7 & 6.0 & 3.2 & 39.2 & 194 \\
\hline 8 & 3.8 & 50.12 & 31.4 & 193 \\
\hline 9 & 0.7 & 3.14 & 39.6 & 197 \\
\hline 10 & 2.9 & ND & 43.9 & 257 \\
\hline 11 & 2.9 & 2.01 & 43.2 & 162 \\
\hline 12 & 0.2 & 10.22 & 31.0 & 173 \\
\hline 13 & 0.7 & 2.71 & 42.3 & 222 \\
\hline 14 & 1.1 & 2.94 & 44.4 & 146 \\
\hline Mean \pm SD & $2.04 \pm 1.72$ & $7.86 \pm 12.94$ & $38.64 \pm 4.26$ & $180.07 \pm 33.74$ \\
\hline
\end{tabular}

CEA, carcinoembryonic antigen; Cyfra21-1, fragments of cytokeratin-19; ALB, albumin; LDH, lactate dehydrogenase; ND, not done; SD, standard deviation.

PET imaging provides a novel non-invasive method to confirm the malignant potential of solitary pulmonary nodules. The sensitivity, specificity and accuracy of PET scans in qualitative diagnosis of solitary pulmonary nodule reaches 90.0, 93.3 and $91.7 \%$, respectively when using $\mathrm{SUV}_{\max } \geq 2.5$ as a diagnostic criterion of malignant nodules (19). PET imaging was used in 4 patients in the present study and all $\mathrm{SUV}_{\max }$ values were $>2.5$. However, Ding et al (20) identified that a patient with primary pulmonary LELC, confirmed by surgery and pathology, received a false-negative result on a PET scan which revealed $\mathrm{SUV}_{\max }<2.5$. Therefore, PET imaging alone has a limited effectiveness in the case of pulmonary LELC.

The association between serum tumor markers and pulmonary LELC remains unknown. A Chinese study (21) identified that the level of CA125 increased significantly in $77.8 \%$ of patients, which suggested that CA125 may be used as an indicator of LELC. In the present study, 1 patient exhibited an increased level of CEA which indicates that CEA may not be associated with LELC. Serum levels of Cyfra21-1 were increased in $55.56 \%$ of patients in the present study and the correlation coefficient of maximum diameter and Cyfra21-1 was $0.696(\mathrm{P}=0.008)$. However, the association between Cyfra21-1 and the onset of LELC remains unknown. Liang et al (22) identified that the levels of serum LDH and serum ALB were normal in patients with early-stage LELC and the serum ALB level was an independent prognostic factor in a Cox's regression model $(\mathrm{P}=0.005)$.

Previous studies have demonstrated that the majority of patients with pulmonary LELC were in early resectable and locally advanced stages $(8,23)$. There is no unified therapeutic method to treat primary LELC of the lung. The National Comprehensive Cancer Network guidelines (24) recommend the use of adjuvant chemotherapy in patients with NSCLC stage between IIA/B and IIIAs in which the tumors have been completely resected. The majority of case studies have suggested that pulmonary LELC is sensitive to chemotherapy. A previous study demonstrated that surgery and adjuvant chemotherapy provided an improved prognosis for patients in early-stage LELC and advanced-stage LELC exhibited an increased sensitivity to chemotherapy and radiotherapy (25). For advanced pulmonary LELC, platinum-based chemotherapy is regarded as the first-line treatment. Adjuvant chemotherapy has been previously identified to markedly improve the prognosis for patients with stage IIIA LELC who underwent complete resection (22). Radiation may be beneficial for the advanced stage of this disease and a radiological dose of between 5,000 and 7,000 cGy is frequently used, depending on tumor location.

Patients with pulmonary LELC exhibited a favorable prognosis compared with that of patients with other types of NSCLC; furthermore, patients with the former achieved increased survival time and improved overall survival (OS) rates under multimodality treatment $(23,25)$. According to a previous study (12), the 2- and 5-year OS rates were 81 and $51 \%$, respectively, in patients who received complete resection or chemotherapy or radiotherapy. The 2- and 5-year OS rates were 88 and $62 \%$, respectively, in an additional study (22), which demonstrated that those who underwent complete resection exhibited significantly improved OS rates $(\mathrm{P}<0.05)$. Patients with locally advanced-stage LELC achieved increased survival rates with multiple therapeutic methods, compared with patients without any treatment (22). The presence of abundant cluster of differentiation-8-positive cytotoxic $\mathrm{T}$ lymphocytes and the underexpression of p53 and c-erbB2 oncogene protein in tumor cells is considered to be evidence for improved prognosis (10). It is well-known that epidermal 
growth factor receptor (EGFR) genes are associated with the pathogenesis of lung cancer; however, the status of EGFRs in LELC remains unknown. Chang et al (26) revealed that EGFR mutation was typically observed in patients with tumor size $\leq 3 \mathrm{~cm}(\mathrm{P}=0.014)$ and EGFR overexpression was more common in female than in male patients. EGFR mutation was not common in LELC; thus, EGFR may not serve an important role in carcinogenesis for this type of lung cancer. Furthermore, it has been hypothesized that EGFR tyrosine kinase inhibitors may be beneficial to those inoperable patients (26).

Lung cancer is the leading cause of cancer-related mortality globally and advanced lung cancer is typically considered to exhibit a poor prognosis. However, pulmonary LELC, a rare subtype of NSCLC, exhibits an improved prognosis under multimodality treatment compared with that of non-LELC (including patients with stage IV). Pulmonary LELC typically affects young and non-smoking patients, is associated with EBV infection and the majority of patients are in early or locally advanced stages. Common treatments of the tumor include surgery, chemotherapy and radiotherapy, depending on the clinical stage of the tumor. Surgery with postoperative chemotherapy-based multimodality treatment is recommended. However, due to the low incidence, the treatment of advanced LELC is controversial and usually depends on clinical experience. It is hypothesized that clinicians, pathologists and radiologists may consider LELC in the differential diagnosis, particularly in an EBV epidemic region. Additional studies are required to optimize the various treatment modalities of the malignant tumor.

\section{Acknowledgements}

The present study was supported by the Key Specialty Discipline Construction Program of Fujian and Nation, P.R.C. The authors thank the surgeon, pathologists, radiologists and nurses of the Fujian Medical University Union Hospital (Fujian, China) for their assistance and coordination in the present study.

\section{References}

1. Wong JF and Teo MC: Case report: Lymphoepithelial-like carcinoma of the lung-a chronic disease? World J Surg Oncol 10: 91, 2012.

2. Williamson SR, Zhang S, Lopez-Beltran A, Shah RB, Montironi R, Tan PH, Wang M, Baldridge LA, MacLennan GT and Cheng L: Lymphoepithelioma-like carcinoma of the urinary bladder: Clinicopathologic, immunohistochemical, and molecular features. Am J Surg Pathol 35: 474-483, 2011.

3. Blasi MA, Ventura L, Laguardia M, Tiberti AC, Sammarco MG and Balestrazzi E: Lymphoepithelioma-like carcinoma involving the lacrimal gland and infiltrating the eyelids. Eur J Ophthalmol 21: 320-323, 2010.

4. Brun JL, Randriambelomanana J, Cherier L, Lafon ME, Trufflandier N and Le Bail B: Lymphoepithelioma-like carcinoma of the ovary: A case report and review of the literature. Int J Gynecol Pathol 29: 427-431, 2010.

5. Tseng CJ, Pao CC, Tseng LH, Chang CT, Lai CH, Soong YK, Hsueh S and Jyu-Jen H: Lymphoepithelioma-like carcinoma of the uterine cervix: Association with Epstein-Barr virus and human papillomavirus. Cancer 80: 91-97, 1997.
6. Aoki R, Mitsui H, Harada K, Kawamura T, Shibagaki N, Tsukamoto K, Murata S and Shimada S: A case of lymphoepithelioma-like carcinoma of the skin associated with Epstein-Barr virus infection. J Am Acad Dermatol 62: 681-684, 2010.

7. Bégin LR, Eskandari J, Joncas J and Panasci L: Epstein-Barr virus related lymphoepithelioma-like carcinoma of lung. J Surg Oncol 36: 280-283, 1987.

8. Ho JC, Wong MP and Lain WK: Lymphoepithelioma-like carcinoma of the lung. Respirology 11: 539-545, 2006.

9. Goldstraw P: New TNM classification: Achievements and hurdles. Transl Lung Cancer Res 2: 264-272, 2013.

10. Chang YL, Wu CT, Shih JY and Lee YC: New aspects in clinicopathologic and oncogene studies of 23 pulmonary lymphoepithelioma-like carcinomas. Am J Surg Pathol 26: 715-723, 2002.

11. Han AJ, Xiong M and Zong YS: Association of Epstein-Barr virus with lymphoepithelioma-like carcinoma of the lung in southern China. Am J Clin Pathol 114: 220-226, 2000.

12. Mo Y, Shen J, Zhang Y, Zheng L, Gao F, Liu L and Xie C: Primary lymphoepithelioma-like carcinoma of the lung: Distinct computed tomography features and associated clinical outcomes. J Thorac Imaging 29: 246-251, 2014.

13. Brambilla E, Pugatch B, Geisinger K, et al: Large cell carcinoma. In: WHO Classification of tumors-pathology \& genetics-tumours of the lung, pleura, thymus and heart. IARC Press, Lyon, 2004.

14. Castro CY, Ostrowski ML, Barrios R, Green LK, Popper HH, Powell S, Cagle PT and Ro JY: Relationship between Epstein-Barr virus and lymphoepithelioma-like carcinoma of the lung: A clinicopathologic study of 6 cases and review of the literature. Hum Pathol 32: 863-872, 2001.

15. Wei WI and Sham JS: Nasopharyngeal carcinoma. Lancet 365: 2041-2054, 2005.

16. Ooi GC, Ho JC, Khong PL, Wong MP, Lam WK and Tsang KW: Computed tomography characteristics of advanced primary pulmonary lymphoepithelioma-like carcinoma. Eur Radiol 13: 522-526, 2003.

17. Hoxworth JM, Hanks DK, Araoz PA, Elicker BM, Reddy GP, Webb WR, Leung JW and Gotway MB: Lymphoepithelioma-like carcinoma of the lung: Radiologic features of an uncommon primary pulmonary neoplasm. AJR Am J Roentgenol 186: 1294-1299, 2006.

18. Ma H, Wu Y, Lin Y, Cai Q, Ma G and Liang Y: Computed tomography characteristics of primary pulmonary lymphoepithelioma-like carcinoma in 41 patients. Eur J Radiol 82: 1343-1346, 2013.

19. Shen DH, Cheng CY, Lin LF, Gao HW, Cheng YL and Chen CY: Conversion from FDG-negative to-positive during follow-up in a rare case of pulmonary lymphoepithelioma-like carcinoma. Clin Nucl Med 37: 679-681, 2012.

20. Ding Q, Hua Y, Guan Y, et al: A comparative study of PET and PET/CT in solitary pulmonary nodules. Chin J Nucl Med 25: 261-263, 2005 (In Chinese).

21. Xia J, Jiang L, Zhang J, Miao Q, Xin Y, Zhong R, Zhao Y and Han B: The clinical analysis of 21 patients with lymphoepithelioma-like carcinoma after operation. Zhongguo Fei Ai Za Zhi 12: 1169-1173, 2009 (In Chinese).

22. Liang Y, Wang L, Zhu Y, Lin Y, Liu H, Rao H, Xu G and Rong T: Primary pulmonary lymphoepithelioma-like carcinoma: Fifty-two patients with long-term follow-up. Cancer 118: 4748-4758, 2012.

23. Han AJ, Xiong $M, G u$ YY, Lin SX and Xiong $M$ : Lymphoepithelioma-like carcinoma of the lung with a better prognosis: A clinicopathologic study of 32 cases. Am J Clin Pathol 115: 841-850, 2001.

24. NCCN clinical practice guidelines in oncology NSCLC.V3.2011. National Comprehensive Cancer Network. https://www.nccn. org/.

25. Huang CJ, Feng AC, Fang YF, Ku WH, Chu NM, Yu CT, Liu CC Lee MY, Hsu LH, Tsai SY, et al: Multimodality treatment and long-term follow-up of the primary pulmonary lymphoepithelioma-like carcinoma. Clin Lung Cancer 13: 359-362, 2012.

26. Chang YL, Wu CT, Shih JY and Lee YC: Unique p53 and epidermal growth factor receptor gene mutation status in 46 pulmonary lymphoepithelioma-like carcinomas. Cancer Sci 102: 282-287, 2011. 